\title{
BeppoSAX and multiwavelength observations of BL Lacertae in 2000
}

\author{
M. Ravasio ${ }^{1}$, G. Tagliaferri ${ }^{1}$, G. Ghisellini ${ }^{1}$, F. Tavecchio ${ }^{1}$, M. Böttcher ${ }^{2}$, and M. Sikora ${ }^{3}$ \\ 1 Osservatorio Astronomico di Brera, Via Bianchi 46, 23807 Merate, Italy \\ 2 Department of Physics and Astronomy, Clippinger 339, Ohio University, Athens, OH 45701, USA \\ 3 Nicolaus Copernicus Astronomical Center, Bartycka 18, 00-716 Warsaw, Poland \\ Received 14 May 2003 / Accepted 18 June 2003
}

\begin{abstract}
We present two BeppoSAX observations of BL Lac (2200+420) as part of a multiwavelength campaign performed in 2000. The source was in different states of activity: in June, the $X$-ray spectrum was faint and hard $(\alpha \sim 0.8)$, with positive residuals towards low energies. In October we detected the highest [2-10] keV flux ever measured for the source. During this observation, the spectrum was soft $(\alpha \sim 1.56)$ up to $10 \mathrm{keV}$, while above this energy a hard component was dominating. The BeppoSAX data are confirmed by simultaneous RXTE short observations. During the first observation the soft X-ray flux was variable on timescales of a few hours, while the hard X-ray flux was almost constant. During the second observation, instead, the soft spectrum displayed an erratic behaviour with large variations (up to factors 3-4) on timescales smaller than 2 hrs. The analysis of the multiwavelength SED of October showed an intriguing feature: the optical and X-ray sections of the SED are misaligned, while in the prevailing standard picture, they are both thought to be produced via synchrotron emission. We suggested four scenarios to account for this discrepancy: a higher than galactic dust-to-gas ratio towards the source, the first detection of bulk Compton emission in the X-ray band, the presence of two synchrotron emitting regions located at different distances from the nucleus, the detection of a Klein-Nishina effect on the synchrotron spectrum. We discuss the favorable and critical points of each scenario, but, at present, we cannot discriminate between them.
\end{abstract}

Key words. BL Lacertae objects: general - X-rays: galaxies - BL Lacertae objects: individual: BL Lacertae (2200+420)

\section{Introduction}

Blazars are radio-loud Active Galactic Nuclei producing variable non-thermal radiation in relativistic jets oriented close to the line of sight: the emission is therefore beamed and Doppler boosted (Blandford \& Rees 1978). They are characterized by a Spectral Energy Distribution (SED) displaying two broad features: the first, extending from radio to $\mathrm{UV} / \mathrm{X}$-ray is usually ascribed to synchrotron emission; the second, ranging from $\mathrm{X}$-ray to $\gamma$-ray, sometimes up to $\mathrm{TeV}$ energies, is attributed to inverse Compton scattering of seed photons by the same population of synchrotron emitting electrons. The seed radiation field could be constituted by the synchrotron photons themselves (SSC model, Maraschi et al. 1992) or by external photons, produced by the accretion disk (Dermer \& Schlickeiser 1993), by the Broad Line Region (Sikora et al. 1994; Ghisellini \& Madau 1996) or by hot dust (Blazejowski et al. 2000; Arbeiter et al. 2002). Different contributions of these fields can explain the observed blazar spectra. The BL Lac subclass is characterized by the absence or weakness of broad emission features and is divided in HBL and LBL (High and Low energy peaked BL Lacs) according to the radio to X-ray flux ratio (Padovani \& Giommi 1995). BL Lac itself

Send offprint requests to: M. Ravasio, e-mail: ravasio@merate.mi.astro.it
(1ES 2200+420) has been classified as a LBL on the basis of its radio-to-X broad band spectral index $\alpha_{\mathrm{rx}}=0.85$ (Sambruna et al. 1996). It was first identified as the optical counterpart of the radio source VRO 42.22.01 by Schmitt (1968); the presence of weak narrow emission lines in its spectrum allowed an accurate measurement of the redshift $z=0.069$ of the host elliptical galaxy (Miller \& Hawley 1977; Miller et al. 1978). It shows superluminal motions on m.a.s. scale $\left(\beta_{\text {app }} \sim 3-4 h^{-1}\right.$, Mutel et al. 1990; $\beta_{\text {app }} \sim 2.2-5.0 h^{-1}$, Denn et al. 2000). In spite of the definition of BL Lac objects as having featureless continua, in 1995, a survey performed by Vermeulen et al. (1995) revealed the presence of an $\mathrm{H} \alpha$ emission line with equivalent width of $7 \AA$, confirmed by subsequent observations (Corbett et al. 1996). During the same year EGRET, aboard the Compton Gamma Ray Observatory, detected a $4.4 \sigma$ excess above $100 \mathrm{MeV}$ from its direction (Catanese et al. 1997). In the summer of 1997, BL Lac entered an exceptional flaring state with the highest X-ray flux ever recorded (Sambruna et al. 1999; Madejski et al. 1999) and a $\gamma$-ray flux 4 times higher than in 1995 (Bloom et al. 1998) .

In the X-ray band, BL Lac has been detected for the first time in 1980 by the IPC $(0.1-4 \mathrm{keV})$ and the MPC (2-10 keV) aboard the Einstein Observatory (Bregman et al. 1990). Since then the source has been observed many times by different satellites such as EXOSAT (Bregman et al. 1990), GINGA 
(Kawai et al. 1991), ROSAT (Urry et al. 1996; Madejski et al. 1999), ASCA (Sambruna et al. 1999; Madejski et al. 1999), RXTE (Madejski et al. 1999) and finally BeppoSAX, which observed it in 1997 (Padovani et al. 2001) and twice in 1999 (Ravasio et al. 2002).

During the second half of 2000, from July to December, the source has been the object of an intensive multiwavelength campaign (Böttcher et al., in prep.) which included two X-ray observations performed by BeppoSAX and was supplemented by a continuous long-term monitoring program by the Rossi $\mathrm{X}$-ray Timing Explorer (RXTE), with 3 short pointings per week (Marscher et al., in prep.).

During this campaign BL Lac has been observed in the radio band by the telescopes of the University of Michigan and of the Metsähovi Radio Observatory, while in the optical band it has been observed almost continuously by 24 telescopes in the context of an extensive WEBT campaign (Villata et al. 2002). Finally, HEGRA set an upper limit of $25 \%$ of the Crab flux above $0.7 \mathrm{TeV}$, after having accumulated a total of $10.5 \mathrm{~h}$ of on-source time during the autumn of 2000 (Mang et al. 2001). In this paper we will analyze in detail the BeppoSAX data of this campaign, comparing them with RXTE simultaneous ones and discussing the spectral and temporal behaviour of BL Lac in the $\mathrm{X}$-ray band and in the whole radio-to-TeV energy range.

\section{BeppoSAX observations}

Thanks to its uniquely wide energy range $(0.1-200 \mathrm{keV})$, the Italian-Dutch satellite BeppoSAX represents an ideal experiment for looking at blazars and expecially objects such as BL Lac, since it can detect the transition between the synchrotron and inverse Compton components of the SED (Tagliaferri et al. 2000; Ravasio et al. 2002). Therefore it allows to compare the simultaneous behaviour of extremely different parts of the emitting electron distribution. Boella et al. (1997 and references therein) report an extensive summary of the mission.

BeppoSAX observed BL Lac (1ES 2200+420) twice during 2000, since the July 26-27 measurements were soon interrupted (on-source time $=1.69 \times 10^{4} \mathrm{~s}$ ). Therefore we were given a second chance and a new observation started in October 31 lasting until November 2, with a duration of $2.49 \times 10^{4} \mathrm{~s}$. In Table 1 we report the exposures and the mean count rates for each BeppoSAX instrument.

We performed our analysis on linearized and cleaned event files available at the BeppoSAX Science Data Center (SDC) online archive (Giommi \& Fiore 1998) using the software contained in the FTOOLS Package (XIMAGE 2.63c, XSELECT 1.4b, XSPEC 10.00) and XRONOS 4.02. Data from MECS2 \& MECS3 were merged by the SDC team in a single event file. Using XSELECT we extracted spectra and light curves from circular regions around the source of 8 and 4 arcmin radius for LECS and MECS, respectively. We extracted event files also from off-source circular regions, in order to monitor the background behaviour during our measurements. Since the LECS and MECS backgrounds are not uniformly distributed across the detectors, after having checked the constancy of the extracted background light curves, we choose to use the background files obtained from long blank field exposures, available at the SDC public ftp site (Fiore et al. 1999; Parmar et al. 1999).

\subsection{Spectral analysis}

The spectral analysis was performed with XSPEC 10.0, using the updated response matrices and blank-sky background files $(01 / 2000)$ available at the SDC public ftp site. The LECS/MECS normalization factor was fixed at 0.72 , while the PDS/MECS was fixed at 0.9 to be consistent with our previous works (Ravasio et al. 2002): these values are within the acceptable range indicated by SDC (LECS: 0.67-1, PDS: 0.77-0.93, Fiore et al. 1999).

\subsubsection{July $26-27$}

During this observation, the source was not detected by PDS because of the short on-source time and because of the intrinsic weakness of the source itself. For the same reasons and because of the high galactic absorption, the detection was uncertain also at LECS low energies: therefore we proceeded to the analysis only in the [0.6-10] keV range, fitting the extracted spectrum with a single power law model.

We repeated the procedure three times, letting the interstellar absorption parameter either free to vary, or fixed at the value $N_{\mathrm{H}}=3.6 \times 10^{21} \mathrm{~cm}^{-2}$. The latter is obtained adding the galactic value from $21 \mathrm{~cm}$ measurements $N_{\mathrm{H}}=(2.0 \pm 1.0) \times 10^{21} \mathrm{~cm}^{-2}$ (Elvis et al. 1989) and the absorption due to the molecular clouds observed along the line of sight (Lucas \& Liszt 1993). Finally, we used the fixed value $N_{\mathrm{H}}=2.5 \times 10^{21} \mathrm{~cm}^{-2}$, to be consistent with previous works (Ravasio et al. 2002; Sambruna et al. 1999; Madejski et al. 1999).

Letting the absorption parameter $N_{\mathrm{H}}$ free, the $0.6-10 \mathrm{keV}$ spectrum is well fitted by a single power law with spectral index $\alpha=0.75 \pm 0.15\left(\chi_{\mathrm{r}}^{2} /\right.$ d.o.f. $\left.=0.96 / 50\right)$. We obtained $N_{\mathrm{H}}=$ $2.0 \pm 1 \times 10^{21} \mathrm{~cm}^{-2}$, the large uncertainties are due to the low count rate in the soft X-ray band.

A single power law model with $N_{\mathrm{H}}$ fixed at $3.6 \times 10^{21} \mathrm{~cm}^{-2}$ leaves large positive residuals below $\sim 1 \mathrm{keV}$. This suggests that we are using an exceedingly high absorption column or that near the low boundary of our energy range we are detecting the transition between a soft and a hard spectral component.

These residuals become less relevant adopting the intermediate value $N_{\mathrm{H}}=2.5 \times 10^{21} \mathrm{~cm}^{-2}$ (Fig. 1): in this case a single power law model with $\alpha_{\mathrm{X}}=0.8 \pm 0.1$ fits the data well $\left(\chi_{\mathrm{r}}^{2} /\right.$ d.o.f. $\left.=0.96 / 51\right)$.

According to an F-test, a broken power law model does not improve significantly the quality of the fit. The best fit parameters for each model are shown in Table 2, which reports also the flux at $1 \mathrm{keV}$ and the integrated flux in the $2-10 \mathrm{keV}$ energy range.

\subsubsection{October $30-$ November 2}

The October observation is more interesting because of the longer duration and the higher state of the source. This allows 
Table 1. Journal of BeppoSAX observations.

\begin{tabular}{ccccccc}
\hline \hline & \multicolumn{2}{c}{ LECS } & \multicolumn{2}{c}{ MECS } & \multicolumn{2}{c}{ PDS } \\
\hline Date & $\begin{array}{c}\text { Exposure } \\
(\mathrm{s})\end{array}$ & $\begin{array}{c}\text { Count rate }^{a} \\
\times 10^{-2} \mathrm{cts} \mathrm{s}^{-1}\end{array}$ & $\begin{array}{c}\text { Exposure } \\
(\mathrm{s})\end{array}$ & $\begin{array}{c}\text { Count rate }^{b} \\
\times 10^{-2} \mathrm{cts} \mathrm{s}^{-1}\end{array}$ & $\begin{array}{c}\text { Exposure } \\
(\mathrm{s})\end{array}$ & $\begin{array}{c}\text { Count rate }^{c} \\
\times 10^{-2} \mathrm{cts} \mathrm{s}^{-1}\end{array}$ \\
\hline $\begin{array}{c}\text { 26-27 July 2000 } \\
\text { 31 October- }\end{array}$ & 16917 & $4.99 \pm 0.20$ & 23309 & $8.23 \pm 0.21$ & $1.04 \times 10^{4}$ & $-1.20 \pm 5.73$ \\
2 November 2000 & 24899 & $28.5 \pm 0.38$ & 33661 & $33.5 \pm 0.33$ & $1.88 \times 10^{4}$ & $12.92 \pm 4.27$ \\
\hline
\end{tabular}

${ }^{a} 0.1-10 \mathrm{keV} ;{ }^{b} 1.5-10 \mathrm{keV} ;{ }^{c} 12-100 \mathrm{keV}$.

Table 2. LECS + MECS spectrum best-fit parameters.

\begin{tabular}{|c|c|c|c|c|c|c|}
\hline \multicolumn{7}{|c|}{ 26-27 July 2000 } \\
\hline $\begin{array}{l}N_{\mathrm{H}} \\
\times 10^{21} \mathrm{~cm}^{-2}\end{array}$ & $\alpha_{1}$ & $\begin{array}{c}E_{\mathrm{b}} \\
\mathrm{keV}\end{array}$ & $\alpha_{2}$ & $\begin{array}{c}F_{1 \mathrm{keV}} \\
\mu \mathrm{Jy}\end{array}$ & $\begin{array}{l}F_{2-10 \mathrm{keV}} \\
\mathrm{erg} \mathrm{cm}^{-2} \mathrm{~s}^{-1}\end{array}$ & $\chi_{\mathrm{r}}^{2} /$ d.o.f. \\
\hline \multicolumn{7}{|l|}{ Free } \\
\hline $2.0 \pm 1$ & $0.75 \pm 0.15$ & & & 1.1 & $5.8 \times 10^{-12}$ & $0.96 / 50$ \\
\hline \multicolumn{7}{|l|}{ Fixed } \\
\hline 3.6 & $0.9 \pm 0.1$ & & & 1.3 & $5.8 \times 10^{-12}$ & $1.07 / 51$ \\
\hline \multicolumn{7}{|l|}{ Fixed } \\
\hline 2.5 & $0.8 \pm 0.1$ & & & 1.1 & $5.8 \times 10^{-12}$ & $0.96 / 51$ \\
\hline \multicolumn{7}{|c|}{31 October-2 November 2000} \\
\hline $\begin{array}{l}N_{\mathrm{H}} \\
\times 10^{21} \mathrm{~cm}^{-2}\end{array}$ & $\alpha_{1}$ & $\begin{array}{c}E_{\mathrm{b}} \\
\mathrm{keV}\end{array}$ & $\alpha_{2}$ & $\begin{array}{c}F_{1 \mathrm{keV}} \\
\mu \mathrm{Jy}\end{array}$ & $\begin{array}{l}F_{2-10 \mathrm{keV}} \\
\mathrm{erg} \mathrm{cm}^{-2} \mathrm{~s}^{-1}\end{array}$ & $\chi_{\mathrm{r}}^{2} /$ d.o.f. \\
\hline \multicolumn{7}{|c|}{ Power law models } \\
\hline \multicolumn{7}{|l|}{ Free } \\
\hline $3.0 \pm 0.3$ & $1.63 \pm 0.06$ & & & 13.0 & $2.1 \times 10^{-11}$ & $0.69 / 76$ \\
\hline \multicolumn{7}{|l|}{ Fixed } \\
\hline 3.6 & $1.71 \pm 0.03$ & & & 14.6 & $2.1 \times 10^{-11}$ & $0.96 / 77$ \\
\hline \multicolumn{7}{|l|}{ Fixed } \\
\hline 2.5 & $1.56 \pm 0.03$ & & & 11.9 & $2.1 \times 10^{-11}$ & $0.87 / 77$ \\
\hline \multicolumn{7}{|c|}{ Broken power law models } \\
\hline Fixed & & & & & & \\
\hline 2.5 & $1.45_{-0.5}^{+0.1}$ & $2.2_{-1.3}^{+0.7}$ & $1.65 \pm 0.08$ & 11.4 & $2.1 \times 10^{-11}$ & $0.68 / 75$ \\
\hline
\end{tabular}

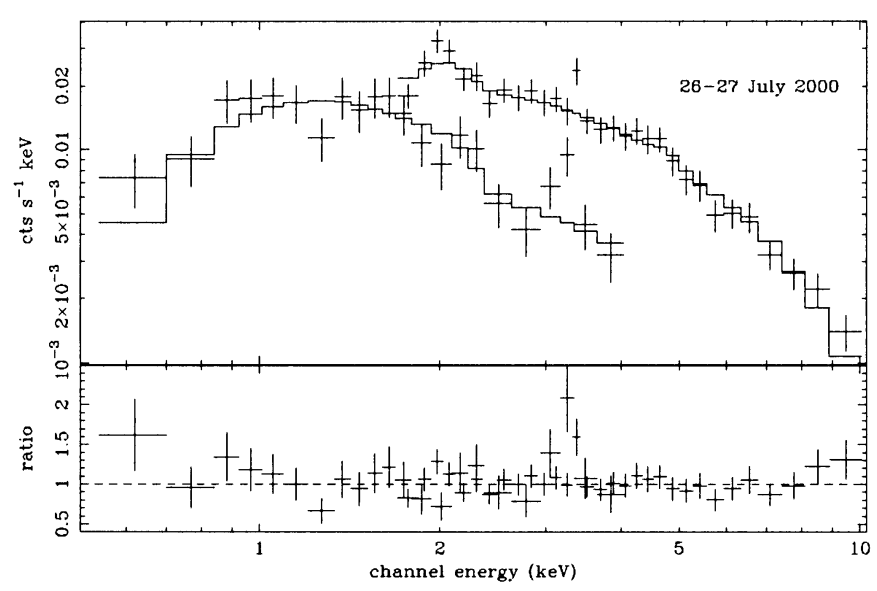

Fig. 1. LECS+MECS 26-27 July spectrum of BL Lac. The spectrum is modeled with a power law and a fixed $N_{\mathrm{H}}=2.5 \times 10^{21} \mathrm{~cm}^{-2}$. We choose this value to be consistent with previous work (Ravasio et al. 2002; Madejski et al. 1999; Sambruna et al. 1999). us to analyze a wider spectral range, from $0.3 \mathrm{keV}$ up to $50 \mathrm{keV}$, thanks to the detection by the PDS.

As before, we performed the fits letting $N_{\mathrm{H}}$ free, then fixing it to its maximum value and finally to $N_{\mathrm{H}}=2.5 \times 10^{21} \mathrm{~cm}^{-2}$. In the first case, the LECS + MECS spectrum was well fitted by a soft single power law model, with energy index $\alpha_{\mathrm{X}}=$ $1.63 \pm 0.06\left(N_{\mathrm{H}}=3.0 \pm 0.3 \times 10^{21} \mathrm{~cm}^{-2}\right)$. A similar result is obtained also keeping $N_{\mathrm{H}}$ fixed to $2.5 \times 10^{21} \mathrm{~cm}^{-2}$, while the model with the highest absorption value leaves positive residuals increasing towards low energies.

We repeated the procedure using a broken power law model and fixing $N_{\mathrm{H}}=2.5 \times 10^{21} \mathrm{~cm}^{-2}$, for consistence with previous works (e.g. Ravasio et al. 2002). The LECS + MECS spectrum in the latter case is well fitted by a convex curve steepening beyond $\sim 2 \mathrm{keV}$. The best-fit parameters of each LECS + MECS spectral model are listed in Table 2. An F-test suggests that the addition of two parameters gives a $99.9 \%$ probability of improving the quality of the fit.

Subsequently we included also PDS data in the analysis. A single power law model leaves large positive residuals 


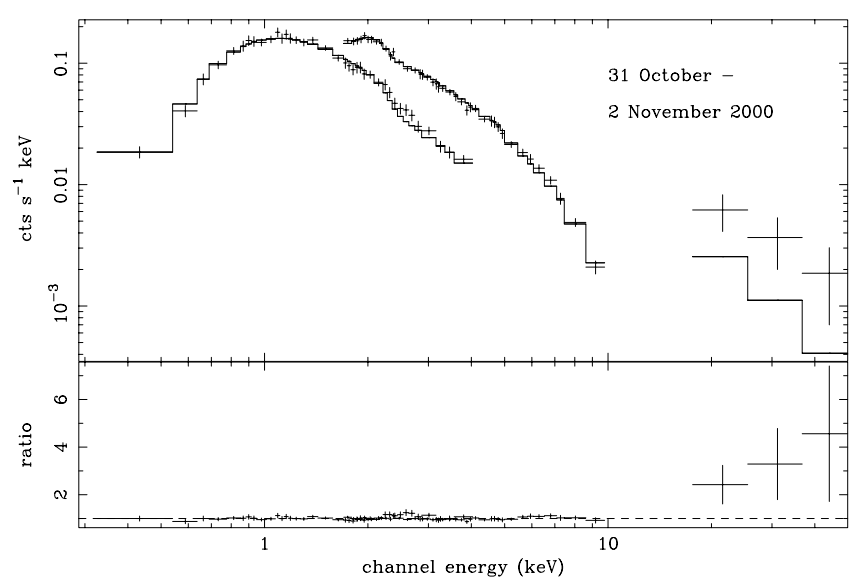

Fig. 2. LECS+MECS+PDS 31 October-2 November spectrum of BL Lac. Both a single and a broken power law model cannot reproduce the whole observed spectrum. PDS data lie above the power-law best fit of the of LECS+MECS spectrum. The LECS+MECS spectrum is well fitted by a convex broken power law.

towards high energies (Fig. 2): the [0.1-50] keV spectrum seems to be concave. PDS data lie largely above the extrapolation of the LECS and MECS spectrum: this can be explained as the rising of a second, hard spectral component. However, the error bars are too large and we cannot constrain a second component in the model. Thus, in order to have an idea of this hard component spectral shape, we fitted the MECS + PDS spectra with a broken power law model, keeping the low energy spectral index fixed to the value obtained from the fit of the LECS + MECS spectrum $(\sim 1.65$, independently from the absorption parameter chosen). The best fit of the two extra parameters are $E_{\mathrm{b}} \sim 9.9 \mathrm{keV}$ and $\alpha_{\mathrm{PDS}} \sim 0.56$, but we are not able to estimate the uncertainties.

\subsubsection{Simultaneous RXTE observations}

In addition to BeppoSAX, the 2000 campaign was covered in the X-ray band also by Rossi X-ray Timing Explorer (Bradt et al. 1993), which provided three short exposures a week, covering the whole duration (Marscher et al., in prep.). We analyzed the two RXTE pointings temporally closest to the BeppoSAX ones. In July the two observations were exactly simultaneous while in November RXTE was lagging BeppoSAX only by $1 \mathrm{hr}$. Therefore we are given the opportunity to test our results with a totally independent set of data.

We restrict our analysis to PCA data (Jahoda et al. 1996), an instrument composed by 5 passively collimated independent $\mathrm{X}$-ray detectors sensitive to the $2-60 \mathrm{keV}$ range.

We choose to compare the [3-15] keV RXTE spectrum to the [1-10] keV MECS using a power law model with fixed absorption parameter $\left(N_{\mathrm{H}}=2.5 \times 10^{21} \mathrm{~cm}^{-2}\right)$. The model parameters are reported in Table 3, together with the log of the observations.

Both RXTE spectra are well fitted by power law models: during the observation of July, RXTE detected a hard spectrum $(\alpha=0.9)$, while in October, it detected a soft component $(\alpha=1.45)$. The slope and the normalization of the

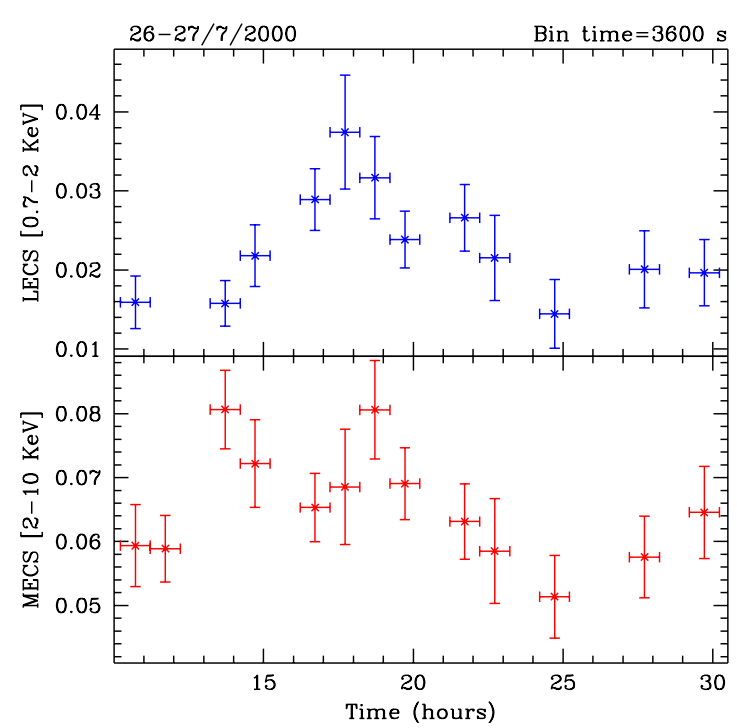

Fig. 3. LECS 0.7-2 keV (top panel), MECS $2-10 \mathrm{keV}$ (bottom panel) light curves of BL Lac during the July 2000 BeppoSAX observation. Note that in the LECS range the source displays larger variability than at higher energies.

best-fit models of RXTE spectra are consistent with those of the MECS, confirming the results obtained from BeppoSAX spectral analysis.

\subsection{Temporal analysis}

We performed this analysis with the XRONOS 5.16 package, grouping data in different time bins to reduce uncertainties. During the observation of July 2000 the source was in a faint state of activity so we choose bins of $3600 \mathrm{~s}$. During the second observation, the source emission was stronger so we could rebin our data in smaller bins of $600 \mathrm{~s}$. In our analysis we excluded the bins with less than $20 \%$ of effective exposure time, to reduce weighting errors.

\subsubsection{6-27 July}

In order to have information on the behaviour of the source at different X-ray wavelenghts we extracted a soft ([0.7-2] keV, LECS) and a hard ([2-10] keV, MECS) X-ray light curve. The source displays large variability in the low energy band: the $0.7-2 \mathrm{keV}$ flux increases by a factor $>2$ on time scales of $\sim 4 \mathrm{hrs}$, then fades to previous values in $\sim 5-6 \mathrm{hrs}$ (Fig. 3 ). In the harder energy band, variability events are less evident but the source is still active, with flux variations of $25-30 \%\left(\chi^{2}\right.$-test constancy probability $\sim 10 \%$ ). The two light curves are only marginally correlated: the null correlation probability is quite high ( 25\%).

This behaviour was noticed also in the June 1999 BeppoSAX observation of BL Lac (Ravasio et al. 2002): in the high energy MECS band (4-10 keV) the source was not variable, while the [0.3-2] keV LECS and the [2-4] keV MECS light curves were displaying a flare of a factor $\sim 4$. 
Table 3. RXTE and BeppoSAX simultaneous observations log and spectral fit parameters. We adopted a power law model with fixed absorption parameter: $N_{\mathrm{H}}=2.5 \times 10^{21} \mathrm{~cm}^{-2}$.

\begin{tabular}{|c|c|c|c|c|c|c|c|}
\hline \multicolumn{8}{|c|}{ 26-27 July } \\
\hline Instrument & Time start & Time end & $\begin{array}{c}\text { Duration } \\
\text { (s) }\end{array}$ & $\bar{\alpha}$ & $\begin{array}{l}F_{1 \mathrm{keV}} \\
(\mu \mathrm{Jy})\end{array}$ & $\begin{array}{l}F_{2-10 \mathrm{keV}}^{*} \\
\left(\times 10^{-12}\right)\end{array}$ & $\overline{\chi_{\mathrm{r}}^{2} / \text { d.o.f. }}$ \\
\hline PCA & $26 / 7 / 00$ & $26 / 7 / 00$ & 2208 & $0.9_{-0.6}^{+0.7}$ & 1.4 & 6.3 & $0.42 / 25$ \\
\hline $3-15 \mathrm{keV}$ & $18: 23: 44$ & 19:00:32 & & & & & \\
\hline MECS & $26 / 7 / 2000$ & $27 / 7 / 2000$ & 23309 & $0.8 \pm 0.1$ & 1.18 & 5.8 & $0.86 / 43$ \\
\hline $1-10 \mathrm{keV}$ & 10:12:39 & 06:43:33 & & & & & \\
\hline \multicolumn{8}{|c|}{30 October-2 November } \\
\hline PCA & $2 / 11 / 2000$ & $2 / 11 / 2000$ & 2000 & $1.45_{-0.25}^{+0.3}$ & 10.3 & 19.7 & $0.45 / 25$ \\
\hline $3-15 \mathrm{keV}$ & $10: 56: 16$ & $11: 29: 36$ & & & & & \\
\hline MECS & $31 / 10 / 2000$ & $2 / 11 / 2000$ & 33661 & $1.6 \pm 0.05$ & 12.7 & 19.7 & $0.61 / 58$ \\
\hline $1-10 \mathrm{keV}$ & $20: 46: 55$ & 09:59:28 & & & & & \\
\hline
\end{tabular}

\subsubsection{October-2 November}

During the October BeppoSAX observation, BL Lac displayed very fast and remarkable flux variations in the whole energy range covered by the LECS and the MECS (Fig. 4). The light curves are characterized by a similar, erratic behaviour, with flux variations of factors $\sim 3-4$, even on timescales of $\sim 1 \mathrm{hr}$. The three light curves are highly correlated $(P>99.9 \%)$ even with a short temporal binning of $10 \mathrm{~min}$.

We analyzed also the PDS light curve, which turned out to be constant: a test gives a $\sim 96 \%$ constancy probability. Because of the large errorbars, however, we would not be able to detect variations smaller than a factor of 3. For BL Lac, this X-ray behaviour is not unprecedented: as already mentioned, during the June 1999 BeppoSAX observation, the [0.3-2] keV flux doubled in $20 \mathrm{~min}$ and faded to previous values in a similar time. In less than $2 \mathrm{hrs}$ a complete flare with a flux variation of a factor $\sim 4$ was observed. The amplitude of this event was highly frequency-dependent: while the flare was extremely prominent in the [0.3-2] keV and in the [2-4] keV curves, at higher frequencies the flux remained constant.

The best way to characterize the temporal behaviour of the source would be the calculation of the power density spectrum: however this tool is not appropriate for unevenly sampled light curves like BeppoSAX ones. The large observational gaps, the short duration of the run and the limited statistics caused by the faintness of the source also makes the use of alternative techniques very difficult, such as the discrete correlation function (Edelson \& Krolik 1988) or the structure function calculation (Simonetti et al. 1985).

We can still characterize the variability degree of the source using two common estimators: the normalized excess variance parameter $\sigma_{\mathrm{rms}}^{2}$ and the minimum doubling timescale $T_{\text {short }}$ (Zhang et al. 1999; Fossati et al. 2000). The first parameter is defined as the normalized difference between the variance of the light curve and the variance due to measurement errors: it quantifies the mean variability of the source. The second parameter, instead, represent a measure of the fastest significant timescale of the source (Edelson 1992). Assuming that each

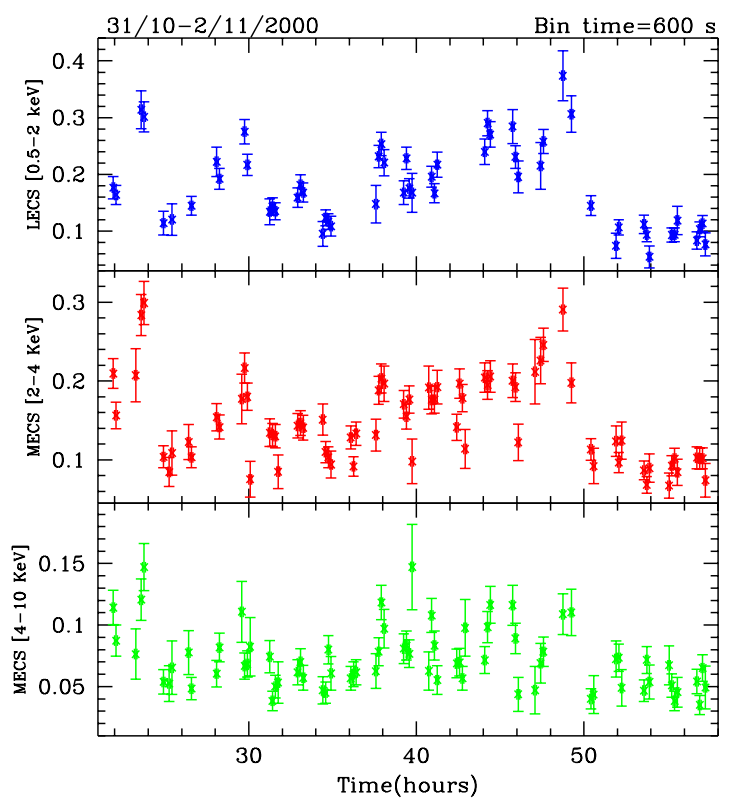

Fig. 4. LECS 0.5-2 keV (top panel), MECS 2-4 keV (mid panel) and MECS 4-10 keV (bottom panel) light curves of BL Lac during the October-November 2000 BeppoSAX observation.

point of the light curve is described as $\left(t_{i}, f_{i}\right)$ we define the "doubling time" as

$T_{i j}=\left|\frac{f_{i j} \Delta T_{i j}}{\Delta f_{i j}}\right|$

where $\Delta T_{i j}=t_{i}-t_{j}, \Delta f_{i j}=f_{i}-f_{j}$ and $f_{i j}=\left(f_{i}+f_{j}\right) / 2$.

For each of the discussed energy ranges ([0.5-2] keVLECS; [2-4] keV-MECS; [4-10] keV-MECS) we produced 4 different light curves with time bin of 500, 1000, 1500 and $2000 \mathrm{~s}$, respectively. For each curve we computed the $\sigma_{\text {rms }}^{2}$ and the average of the 5 smallest $T_{i j}$ (calculated for each data pair $(i, j)$ of the light curve) with fractional error lower than $25 \%$. We define $T_{\text {short }}$ as their weighted average. In Table 4 we summarize our results. 
Table 4. Variability parameters.

\begin{tabular}{lcccc}
\hline \hline \multicolumn{5}{c}{$\mathbf{3 0 / 1 0 - 2 / 1 1 ~ 2 0 0 0}$} \\
\hline $\begin{array}{l}\text { Energy band } \\
(\mathrm{keV})\end{array}$ & $\begin{array}{c}<\text { Count rate }^{a} \\
\left(10^{-2} \mathrm{cts} / \mathrm{s}\right)\end{array}$ & $\begin{array}{c}\sigma_{\text {rms }}^{2}{ }^{a} \\
\left(10^{-2}\right)\end{array}$ & $\begin{array}{c}<\sigma_{\text {rms }}^{2}>^{b} \\
\left(10^{-2}\right)\end{array}$ & $\begin{array}{c}T_{\text {short }}^{b} \\
(\mathrm{ks})\end{array}$ \\
\hline LECS & 17.6 & 15.2 & 12.8 & 6.3 \\
$0.5-2 \mathrm{keV}$ & \pm 7.2 & \pm 3.3 & \pm 1.6 & \pm 0.6 \\
\hline MECS & 14.7 & 11.4 & 10.1 & 5.5 \\
$2-4 \mathrm{keV}$ & \pm 5.3 & \pm 2.5 & \pm 1.5 & \pm 0.5 \\
\hline MECS & 7.4 & 7.9 & 7.5 & 7.1 \\
$4-10 \mathrm{keV}$ & \pm 2.5 & \pm 2.1 & \pm 1.1 & \pm 1.3 \\
\hline
\end{tabular}

${ }^{a}$ From $600 \mathrm{~s}$ rebinned light curves.

${ }^{b}$ Weighted mean of data taken from 500,1000, 1500, $2000 \mathrm{~s}$

rebinned light curves.

The highest frequency light curve is the least variable, as can be noticed also by looking at Fig. 4: probably this can be explained partially as an effect of the larger measurement error caused by poorer statistics and partially as a lower variability at higher energies.

Within the relatively large errors, no significant differences are found between the $T_{\text {short }}$ values of the three light curves: the source seems to have a characteristic minimum variability timescale of $\sim 1.5-2 \mathrm{hrs}$.

\section{Spectral Energy Distributions}

After having performed the spectral analysis we are now able to build the SEDs of BL Lac relative to the BeppoSAX 2000 observations and to compare them with other historical multiwavelength SEDs.

As we have discussed above, during 2000 BeppoSAX detected the source in two completely different states of activity: in July the source was detected only in the [0.6-10] keV range, displaying a faint $\left(F_{2-10}=5.8 \times 10^{-12} \mathrm{erg} \mathrm{cm}^{-2} \mathrm{~s}^{-1}\right)$ hard spectrum. In October-November, instead, BL Lac was displaying a very intense $\left(F_{2-10}=2.1 \times 10^{-11} \mathrm{erg} \mathrm{cm}^{-2} \mathrm{~s}^{-1}\right)$ soft spectrum up to $\sim 10 \mathrm{keV}$, while at higher energies a hard component, detected by the PDS up to $45 \mathrm{keV}$, was dominant.

Also in the optical band the source was in different states: the increase of the optical flux, in fact, was the reason for prolonging the multiwavelength campaign beyond August 2000. The optical fluxes measured simultaneously to the BeppoSAX observations differed by $40 \%$ between the two pointings: in the core of the campaign (26-27 July) the source average $R$-band magnitude was $m_{R}=14.08$, while during the second X-ray run it was $m_{R}=13.74$ (Villata et al. 2002). After having dereddened the data using $A_{B}=1.42$ (Schlegel et al. 1998), we calculated the optical spectral indices using weight-averaged $U B V R I$ fluxes (see values in Fig. 5 caption): when fainter, the spectrum is softer $\left(\alpha_{\text {opt }} \sim 1.84 \pm 0.01\right)$, while during the autumn it is harder $\left(\alpha_{\mathrm{opt}} \sim 1.58 \pm 0.04\right)$.

In Fig. 5 we plot the two simultaneous multiwavelength SEDs of BL Lac. We report also the upper limit by HEGRA, $7.7 \times 10^{-12}$ photons $\mathrm{cm}^{-2} \mathrm{~s}^{-1}$ above $0.7 \mathrm{TeV}$ (Mang et al. 2001), which is $25 \%$ of the Crab level: this means $F_{0.7 \mathrm{TeV}}=$ $5.13 \times 10^{-15} \times\left(\alpha_{\mathrm{TeV}}\right)$ Jy, where $\alpha_{\mathrm{TeV}}$ is the energy index of the $\mathrm{TeV}$ spectrum (assumed to be $=1.5$ in the plot).

\subsection{Historical SEDs}

As mentioned in the introduction, blazar SEDs are characterized by two broad humps extending, the first, from radio to $\mathrm{UV} / \mathrm{X}$-rays and the second from $\mathrm{X}$-rays to $\gamma$-ray or even TeV energies. The first component is usually interpreted as synchrotron emission from high-energy electrons in relativistic motion, while the second is explained as inverse Compton scattering of seed photons of still debated origin.

The multiwavelength history of BL Lac follows that of its X-ray observations which can be traced back to 1980, the year of the first X-ray detection, carried out by Einstein (Bregman et al. 1990). BL Lac was then observed also by EXOSAT (Bregman et al. 1990), GINGA (Kawai et al. 1991), ROSAT (Urry et al. 1996; Madejski et al. 1999), ASCA (Sambruna et al. 1999; Madejski et al. 1999), RXTE (Madejski et al. 1999) and by BeppoSAX (Padovani et al. 2001; Ravasio et al. 2002).

In Table 5 we report the published spectral parameters for each X-ray observation, together with simultaneous optical ones. The X-ray spectra of BL Lac exhibit a variety of different shapes: sometimes they are concave, hardening towards high energies, and sometimes they are hard in the whole X-ray band. In 1980, IPC ([0.1-4] keV) and MPC ([2-10] keV) aboard Einstein detected quite different spectral indices $\left(\alpha_{1}=2.2\right.$; $\alpha_{2}=0.8$; Bregman et al. 1990) and a similar shape was detected also by ASCA in November 1995 (Sambruna et al. 1999) and by BeppoSAX in June 1999 (Ravasio et al. 2002), as well as during this campaign. In other epochs the X-ray spectrum of BL Lac was displaying only a hard component. In June and July 1988 GINGA detected faint X-ray spectra which can be attributed to inverse Compton emission in a leptonic jet model (Kawai et al. 1991), similarly to the observation of ROSAT (1982), to those performed by BeppoSAX in November 1997 (Padovani et al. 2001), December 1999 (Ravasio et al. 2002) and to our observation of July 2000. All the hard X-ray spectra are similar to each other except for one case: during July 1997 BL Lac was in a high state, consistent with Compton emission, with a 2-10 keV flux $\sim 3-4$ times higher than that observed in 1995 by ASCA or in June 1999 by BeppoSAX; the high state was clearly detected also in the $\gamma$-ray by EGRET (Bloom et al. 1997). The complexity of the behaviour of the source, suggested by the data reported in Table 5 , is further highlighted by the comparison of the multiwavelength SEDs: in Fig. 6 we plot the results of all the multiwavelength campaigns performed on BL Lac.

As evidenced in Figs. 5 and 6 and in Table 5, while during July 2000 we observed the source in a normal state, in the end of October BL Lac was very active. During this run, in fact, BeppoSAX detected the highest soft $\mathrm{X}$-ray flux and an integrated [2-10] keV flux which is only slightly smaller than that of the flare of July 1997, when BL Lac was displaying a hard $\mathrm{X}$-ray spectrum.

\section{Discussion}

X-ray observations are crucial for blazar study because at these energies we can often observe the transition between the two 


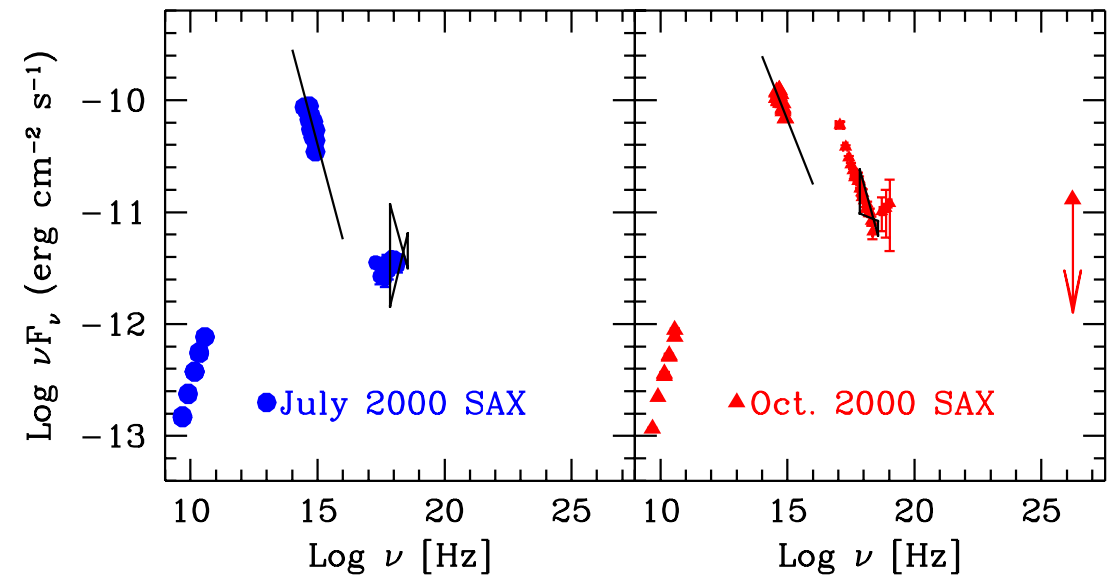

Fig. 5. Left panel: 26-27 July SED. Right panel: 31 October-2 November SED. The black butterflies represent RXTE simultaneous data. The HEGRA upper limit is calculated assuming a spectral energy index $\alpha_{\mathrm{TeV}}=1.5$ above $0.7 \mathrm{TeV}$. The optical slopes are calculated on the weigthed average $U B V R I$ fluxes $\left(A_{B}=1.42\right.$ ). July fluxes (mJy): $\left.\left\langle F_{I}\right\rangle=5.17 \pm 0.07 ;<F_{R}>=8.12 \pm 0.01 ;<F_{V}\right\rangle=12.08 \pm 0.04 ;<F_{B}>=$ $16.54 \pm 0.02 ;\left\langle F_{U}\right\rangle=22.28 \pm 0.06$. October fluxes (mJy): $\left.\left.\left.\left\langle F_{I}\right\rangle=8.26 \pm 0.09 ;<F_{R}\right\rangle=11.96 \pm 0.05 ;<F_{V}\right\rangle=17.56 \pm 0.06 ;<F_{B}\right\rangle=$ $22.43 \pm 0.08 ;<F_{U}>=27.97 \pm 0.18$.

Table 5. June 1980 Einstein data: Bregman et al. (1980). December 1983 EXOSAT data: Bregman et al. (1990). June 1988-July 1988 GINGA data: Kawai et al. (1991). December 1992 ROSAT data: Urry et al. (1992). November 1995 ASCA data: Sambruna et al. (1999). July 1997 RXTE data: Madejski et al. (1999). November 1997 BeppoSAX data: Padovani et al. (2001). June and December 1999 BeppoSAX data: Ravasio et al. (2002).

\begin{tabular}{lccccc}
\hline \hline Date & $\alpha_{1 \mathrm{keV}}$ & $\begin{array}{c}F_{1 \mathrm{keV}} \\
(\mu \mathrm{Jy})\end{array}$ & $\begin{array}{c}F_{2-10 \mathrm{keV}} \\
\left(10^{-12} \mathrm{c.g.s.}\right)\end{array}$ & $\alpha_{\text {opt }}$ & $\begin{array}{c}F_{V} \\
(\mathrm{mJy})\end{array}$ \\
\hline June 1980 & $2.2 \pm 1.0$ & 1.6 & $7.1^{*}$ & $2.2 \pm 0.2$ & 5.75 \\
December 1983 & $(2.2)$ & 0.8 & & $2.1 \pm 0.4$ & 9.55 \\
June 1988 & $0.71 \pm 0.07$ & 1.27 & $7.7^{*}$ & $2.2 \pm 0.1$ & 4.2 \\
July 1988 & $1.16 \pm 0.24$ & 1.35 & $4.1^{*}$ & $3.21 \pm 0.02$ & 6.46 \\
December 1992 & $0.94 \pm 0.46$ & 0.88 & $3.7^{*}$ & & \\
November 1995 & $0.96 \pm 0.04$ & 2.22 & 8.9 & $0.8 \pm 0.2$ & 5.7 \\
July 1997 & $0.59 \pm 0.03$ & 3.55 & 26 & $1.85 \pm 0.4$ & 27.9 \\
November 1997 & $0.89 \pm 0.13$ & 2.4 & 11.2 & & \\
June 1999 & $1.57 \pm 0.25$ & 0.77 & 6.5 & $1.2 \pm 0.2$ & 26.4 \\
December 1999 & $0.63 \pm 0.06$ & 0.96 & 12.3 & $1.8 \pm 0.3$ & 18.6 \\
July 2000 & $0.8 \pm 0.1$ & 1.1 & 5.8 & $1.84 \pm 0.01^{a}$ & $12.08^{a}$ \\
& $0.8 \pm 0.1$ & 1.1 & 5.8 & $0.94 \pm 0.01^{b}$ & $25.60^{b}$ \\
October & $1.45_{-0.5}^{+0.1}$ & 11.4 & 21 & $1.58 \pm 0.04^{a}$ & $17.56^{a}$ \\
November 2000 & $1.45_{-0.5}^{+0.1}$ & 11.4 & 21 & $0.64 \pm 0.02^{b}$ & $37.23^{b}$ \\
\hline
\end{tabular}

${ }^{*}$ From our calculations.

${ }^{a}$ Using $A_{B}=1.42 .{ }^{b}$ Using $A_{B}=2.5$ : see discussion.

emission mechanisms. The two X-ray observations that we presented here clearly show this:

- 26-27 July: during this observation BeppoSAX detected a hard spectrum in the LECS-MECS range, with large positive residuals below $1 \mathrm{keV}$. The hard component can be attributed to inverse Compton emission while the residuals can be explained as the very soft tail of the synchrotron component (the data however does not allow us to set limits on the spectral index of this component or to assess its consistency with the optical data). This picture is supported by the temporal behaviour of the source: the [2-10] keV light curve represents the inverse Compton spectrum produced by the less energetic particles and is therefore almost constant on the timescale of a single observation. The
[0.7-2] keV curve, instead, is influenced also by the soft synchrotron emission produced by highly energetic electrons which can account for the observed variability.

- 31 October-2 November: also in this observation BeppoSAX detected the transition between two emission mechanisms. The LECS+MECS spectrum is soft, while the PDS observed a hard component. In the usual scenario, the hard component is attributed to inverse Compton emission, while the soft spectrum is explained as synchrotron emission by very energetic electrons $\left(\gamma \sim 2.5 \times 10^{4}-10^{5}\right.$ $\left.(B / 1 \mathrm{G})^{-1 / 2}(\delta / 10)^{-1 / 2}\right)$ which have a very short cooling time $\left(t_{\text {syn }} \sim 3 \times 10^{3}-10^{4}(B / 1 G)^{-3 / 2}(\delta / 10)^{1 / 2} \mathrm{~s}\right)$. This could also explain the very fast variability detected in the whole LECS-MECS energy range. 


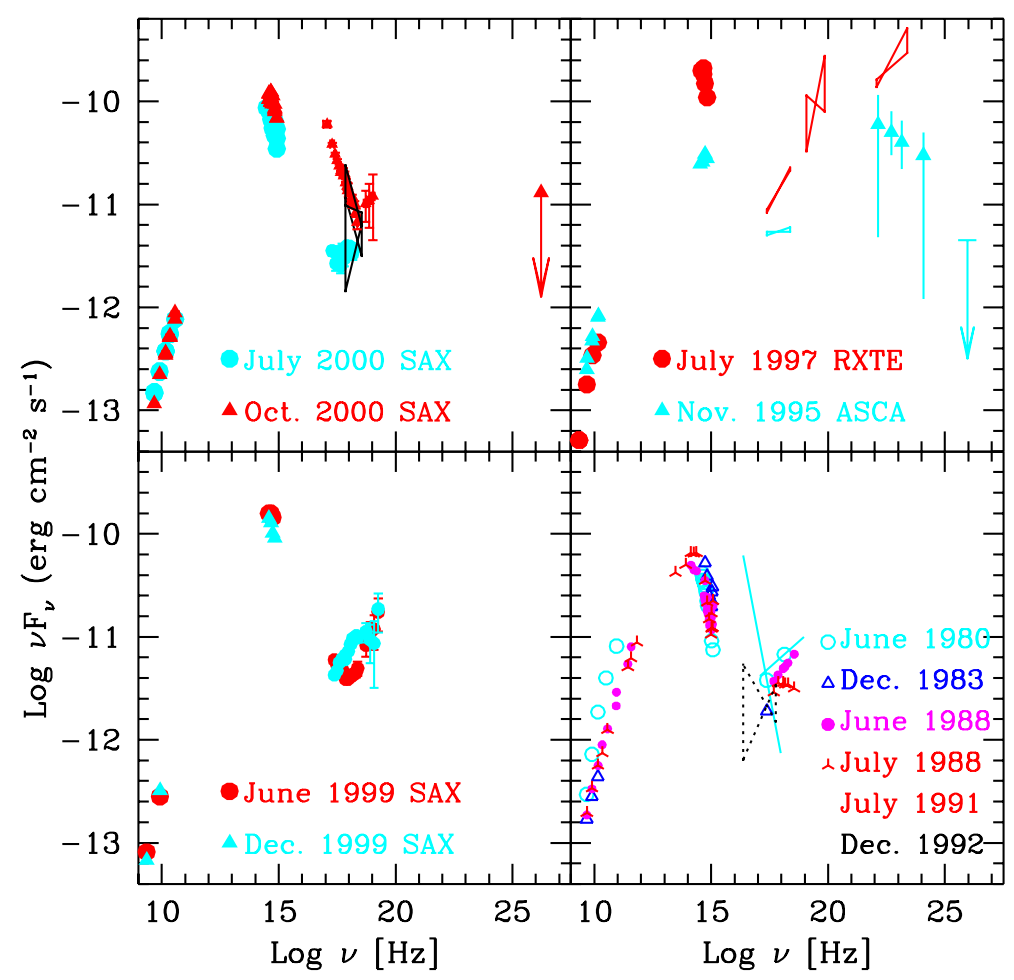

Fig. 6. Simultaneous multiwavelength SEDs of BL Lac. Left-top panel: July and October-November 2000 SEDs (BeppoSAX). We have reported optical data corrected for $A_{B}=1.42$, to be consistent with the other historical observations. Right-top panel: November 1995 (ASCA) and July 1997 (RXTE) SEDs. Left-bottom panel: June and December 1999 SEDs (BeppoSAX). Right-bottom panel: June 1980 (Einstein), December 1983 (EXOSAT), June and July 1988 (GINGA) multiwavelength SEDs. The dotted butterfly represent the December 1982 ROSAT data.

This scenario, however, could be inadequate. Looking at Fig. 5, one can notice immediately a strange feature in the SED of October-November: the X-ray data lie above the extrapolation of the optical spectrum, while both of them should be produced by the same emission mechanism, the synchrotron. Even correcting for the host galaxy contribution (which is almost negligible for the level of activity of BL Lac during our observations) we are not able to reconcile X-ray and optical data. However, we can conceive at least four scenarios which under certain conditions can explain the observed spectral "glitch". They are: i) a variable local absorbing column along the line of sight; ii) bulk Compton radiation; iii) two different synchrotron emitting regions; iv) Klein-Nishina effect on the synchrotron spectrum.

\subsection{Differential absorption}

From the BeppoSAX X-ray analysis we had $N_{\mathrm{H}}=2.5 \times$ $10^{21} \mathrm{~cm}^{-2}$, consistent with previous observations: using the dust-to-gas ratio suggested by Ryter (1996)

$A_{V}=4.5 \times 10^{-22} N_{\mathrm{H}} \mathrm{cm}^{-2}$ and using $E(B-V)=0.329$ as indicated by the NED, the corresponding optical absorption is $A_{B}=1.45$, very similar to the value reported by Schlegel et al. (1998), $A_{B}=1.42$. However, using these values, the $\mathrm{X}$-ray spectrum lies largely above the optical spectrum extrapolation, as illustrated in Fig. 7. Even correcting the optical data for the host galaxy contribution the two sections do not connect continuously. In Fig. 7 we plotted the $R$ and $B$ band BL Lac fluxes corrected using $R_{\text {host }}=15.55$ and $B_{\text {host }}=17.15$ (Villata et al. 2002): the misalignment is even worsened.

If we try to account for this discrepancy using different absorption values, e.g. $A_{B}=2.5$, and consequently $N_{\mathrm{H}}=$ $4.8 \times 10^{21} \mathrm{~cm}^{-2}$, we still obtain two spectral sections which can not be smoothly connected (Fig. 7).

Therefore we fixed the X-ray absorption to $N_{\mathrm{H}}=2.5 \times$ $10^{21} \mathrm{~cm}^{-2}$ and varied the optical absorption.

Using $A_{B} \sim 4.2, U$-filter data lie on the X-ray extrapolation, but the optical spectrum would be very hard and the connection discontinuous. Using an intermediate value, $A_{B}=$ 2.5 , the optical and X-ray spectra connect continuously on a parabolic curve, evidenced in Fig. 7. With this absorption value, we obtain a hard optical spectrum $\left(\alpha_{\mathrm{Jul}}=0.94 \pm 0.01\right.$ and $\alpha_{\text {Oct }}=0.64 \pm 0.02$, for the summer and the autumn 


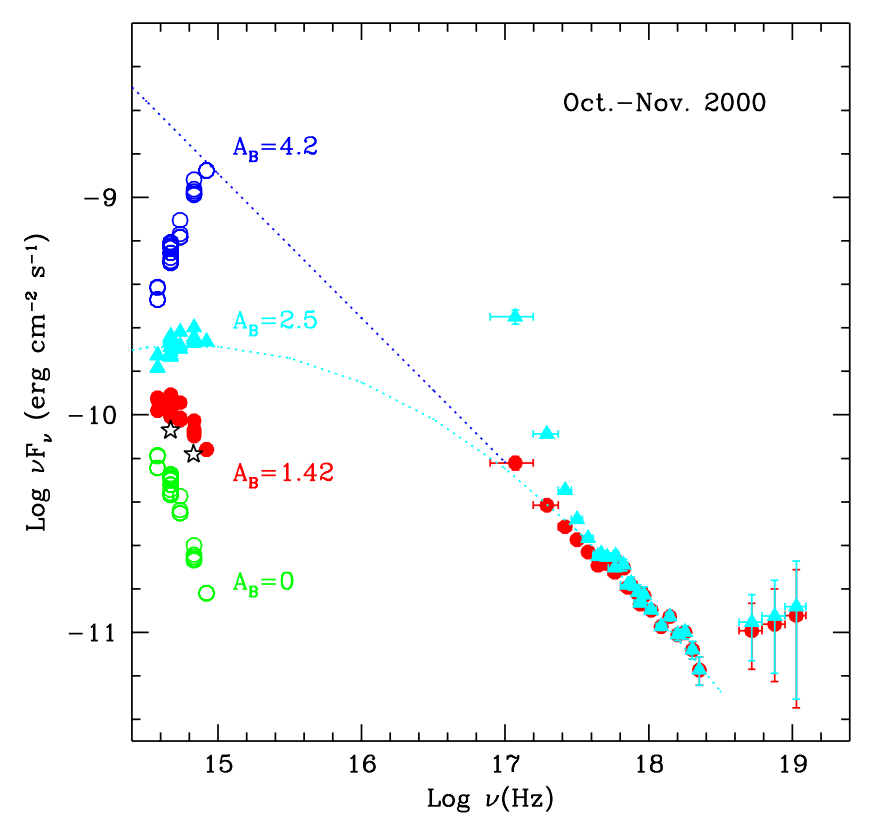

Fig. 7. Optical to X-ray SED of October-November 2000 BeppoSAX observation. Filled circles represent data corrected for $A_{B}=1.42$ corresponding to $N_{\mathrm{H}}=2.5 \times 10^{21} \mathrm{~cm}^{-2}$. Filled triangles represent data corrected for $A_{B}=2.5 \equiv N_{\mathrm{H}}=4.8 \times 10^{21} \mathrm{~cm}^{-2}$. Open circles represent raw optical data and optical data dereddened with $A_{B}=4.2$. The two stars represent the $B$ and $R$-band data dereddened with $A_{B}=1.42$ and corrected for the host galaxy contribution. The two dotted lines represent the extrapolation of the BeppoSAX spectrum to the optical band and a parabolic curve which connects X-ray and optical data dereddened with $A_{B}=2.5$. See text for discussion.

observations respectively) and consequently higher synchrotron peak frequencies. A dust-to-gas ratio higher than that reported by Ryter (1996) could therefore account for the observed optical to $\mathrm{X}$-ray misalignment.

We can compare this behaviour with other multiwavelength campaigns: in Fig. 6 we plot all the published SEDs of BL Lacertae. Only in the SED of 1980 and in that of June 1999 we observe synchrotron emission both in the optical and in the X-ray band. In all the other cases, only the Compton component was detected, thus one cannot see any misalignment between the optical and X-ray synchrotron components. While the BeppoSAX synchrotron spectrum of June 1999 connects continuously with the simultaneous optical data (using the galactic dust-to-gas ratio; Ravasio et al. 2002), in the 1980 SED the optical-UV data seem to lie below the extrapolation of the Einstein IPC spectrum (Bregman et al. 1990), recreating the misalignment observed in October-November 2000.

During the 1980 IPC observation, in fact, BL Lac was displaying a very steep synchrotron spectrum in the [0.1-4] keV range ( $\alpha=2.2 \pm 1.0$, using however a very high absorption parameter $N_{\mathrm{H}}=1.25 \times 10^{22} \mathrm{~cm}^{-2}$; Bregman et al. 1990), hardly connectable with the very soft simultaneous optical-UV spectrum. The Einstein data, however, are affected by very large uncertainties, which make it difficult to determine the exact shape of the SED: this is evidenced by the results published by Worrall \& Wilkes (1990), which reported an IPC spectral index $\alpha=1.34_{-1.3}^{+2.5}$. The uncertainties on the IPC spectral index are such that no firm conclusion about the reality of the opticalto-X-ray misalignment in the 1980 SED can be drawn.

The strange optical/X-ray misalignment observed in 2000 (and maybe in 1980) is not detected in the only other multiwavelength campaign that shows synchrotron emission in the optical and X-ray bands (June 1999, BeppoSAX). Nevertheless, we can rely on the goodness of our data: the BeppoSAX spectrum is confirmed by simultaneous RXTE data, as described in the previous sections, while the optical data are confirmed by different observatories (Villata et al. 2002). The optical/X-ray misalignment is therefore real. The absorption along the line of sight cannot account for it, unless we assume a sudden, large increase of the dust-to-gas ratio in the interstellar material.

This hypothesis, however, is not unlikely, since the line of sight towards BL Lac is partially covered by a low surface brightness interstellar nebulosity which is very variable (Penston \& Penston 1973; Sillanpää, priv. comm.). If these clouds are dusty, they could account for the dust-to-gas ratio excess needed to reconcile the optical and X-ray data in the SED of October-November 2000 (and possibly also in the 1980 SED; Sillanpää et al. 1993). Their proper motion could indeed explain the misalignment in the SED of autumn 2000 and its absence in that of June 1999.

\subsection{Bulk Compton radiation}

Alternatively, the observed misalignment could be claimed as the first detection of the so called "bulk Compton" emission.

This possibility was predicted by Sikora et al. (1997): they postulated the existence of a population of "cold" electrons in addition to the relativistic ones producing synchrotron and inverse Compton emission. These cold electrons, however, have bulk relativistic motion with respect to the radiation fields produced by the accretion disk and by the broad line region and can inverse Compton scatter these photons up to frequencies

$v_{\mathrm{BC}} \simeq \Gamma^{2} v_{0}$

where $v_{0}$ is a characteristic frequency of the external radiation field and $\Gamma$ is the bulk Lorentz factor of the jet. If the seed radiation peaks in the UV band, as the accretion disk and the broad line region emission do, the bulk Compton component will peak in the soft X-ray band accounting for the misalignment we observe in the 2000 BL Lac SED.

Assuming a conical jet with opening angle $\theta_{\mathrm{j}} \sim 1 / \Gamma$ and the conservation of the flux of electrons along the jet $\left(n_{\mathrm{e}} \propto\right.$ $\left.1 / r^{2}\right)$, Sikora et al. (1997) estimated the observed amount of bulk Compton radiation $L_{\mathrm{BC}}$ :

$L_{\mathrm{BC}} \sim 2 \Gamma^{2} \frac{n_{\mathrm{e}}\left(r_{\min }\right) r_{\min } \sigma_{T} \zeta L_{\mathrm{UV}}}{4}$

where $r_{\min }$ is the distance from the apex of the cone at which the jet is fully developed, $L_{\mathrm{UV}}$ is the external radiation field and $\zeta=\zeta(r)$ is the fraction of the UV radiation contributing to the isotropic field at a distance $r$ from the jet apex (Sikora et al. 1997).

In October-November the $\mathrm{X}$-ray luminosity was $L_{\mathrm{X}}=$ $10^{45} \mathrm{erg} \mathrm{s}^{-1}$. Assuming that the bulk Compton emission peaks at $v=10^{17} \mathrm{~Hz}$ and $v_{0}=10^{15} \mathrm{~Hz}$, from Eq. (3) we have $\Gamma \sim 10$. 
Since Vermeulen et al. (1995) observed broad $\mathrm{H} \alpha$ and $\mathrm{H} \beta$ emission lines (confirmed by Corbett et al. 2000), from their data we can evaluate $L_{U V}$ in an indirect way. Postulating a fixed line ratio and following the method described in Celotti et al. (1997) we obtain an average value $L_{\mathrm{BLR}} \sim 5 \times 10^{42} \mathrm{erg} \mathrm{s}^{-1}$. If the Broad Line Region covering factor is $\sim 10 \%$, the disk luminosity is $L_{\mathrm{UV}} \sim 5 \times 10^{43} \mathrm{erg} \mathrm{s}^{-1}$.

Using these values and assuming $L_{\mathrm{BC}}=L_{\mathrm{X}}$ we obtain the particle number density in the observer frame

$n_{\mathrm{e}}\left(r_{\min }\right) \simeq \frac{6 \times 10^{9}}{\Gamma_{10}^{2} \zeta_{0.1} r_{\min , 15}} \mathrm{~cm}^{-3}$

where $\Gamma_{10}=\Gamma / 10, \zeta_{0.1}=\zeta / 0.1$ and $r_{\min , 15}=r_{\min } / 10^{15} \mathrm{~cm}$.

This number particle density, necessary to produce the observed X-ray spectrum, puts constraints on the jet composition. In fact, if we suppose a one-to-one proton-electron plasma, we can easily evaluate the total kinetic power of the jet (Celotti \& Fabian 1993):

$$
\begin{aligned}
L_{\mathrm{KIN}} & \sim \pi\left(r_{\min } \theta_{j}\right)^{2} n_{\mathrm{p}}^{\prime} m_{\mathrm{p}} c^{2} \Gamma^{2} c \\
& \sim 10^{47} \frac{r_{\mathrm{min}, 15} \theta_{j, 0.1}^{2}}{\Gamma_{10} \zeta_{0.1}} \mathrm{erg} \mathrm{s}^{-1}
\end{aligned}
$$

where $n_{\mathrm{p}}^{\prime}=n_{\mathrm{p}} / \Gamma$ is the proton number density in the jet comoving frame. This value exceeds the $L_{\mathrm{KIN}}$ estimated by Celotti et al. (1997) by a factor $\sim 30$. To produce the observed X-ray spectrum via bulk Compton mechanism and to be consistent with the result of Celotti et al. (1997) the proton-electron ratio must be smaller: the jet must be pair loaded.

This is not forbidden since the optical thickness for Thomson scattering $\left(\tau(r)=n_{\mathrm{e}} \sigma_{\mathrm{T}} \theta_{j} r\right)$ at $r_{\min }$ is

$$
\tau\left(r_{\min }\right)=\frac{2}{5} \frac{\theta_{j, 0.1}}{\Gamma_{10}^{2} \zeta_{0.1}}
$$

Since from our calculations $\tau\left(r_{\min }\right)<1$ (and decreasing further out $)$ and the expansion timescale at $r_{\min }\left(t_{\exp }^{\prime} \sim 8 \times\right.$ $10^{4} r_{\min , 15} \Gamma_{10}$ s) is smaller than the annihilation timescale $\left(t_{\text {ann }} \sim 2 \times 10^{5} \Gamma_{10}^{3} \zeta_{0.1} r_{\text {min,15 }} \mathrm{s}\right)$ the pairs will survive along the jet.

After having ruled out the hypothesis of a proton-electron jet and having requested the presence of pairs in the jet, it is now fundamental to understand if the particle number necessary to produce the bulk Compton emission is sufficient to produce the observed SEDs. The particle number conservation we have just demonstrated implies that $n_{\mathrm{e}} \propto 1 / r^{2}$. Therefore, at the distance where the radiation is emitted, $r_{\mathrm{SED}} \sim 10^{16}-10^{17} \mathrm{~cm}$, the particle number density would be

$$
\begin{aligned}
n\left(r_{\mathrm{SED}}\right) & =n\left(r_{\min }\right)\left(\frac{r_{\mathrm{min}}}{r_{\mathrm{SED}}}\right)^{2} \\
& \sim 6 \times 10^{7} \frac{1}{\Gamma_{10}^{2} \zeta_{0.1}} \frac{r_{\mathrm{min}, 15}}{r_{\mathrm{SED}, 17}^{2}} \mathrm{~cm}^{-3} .
\end{aligned}
$$

This value is greater than what we needed to model old BL Lac seds: $n_{\mathrm{e}} \sim 10^{7} / \gamma_{\min } \mathrm{cm}^{-3}$. Only a fraction of the electrons carried by the jet need to be accelerated in order to produce the observed radio to $\gamma$-ray emission.

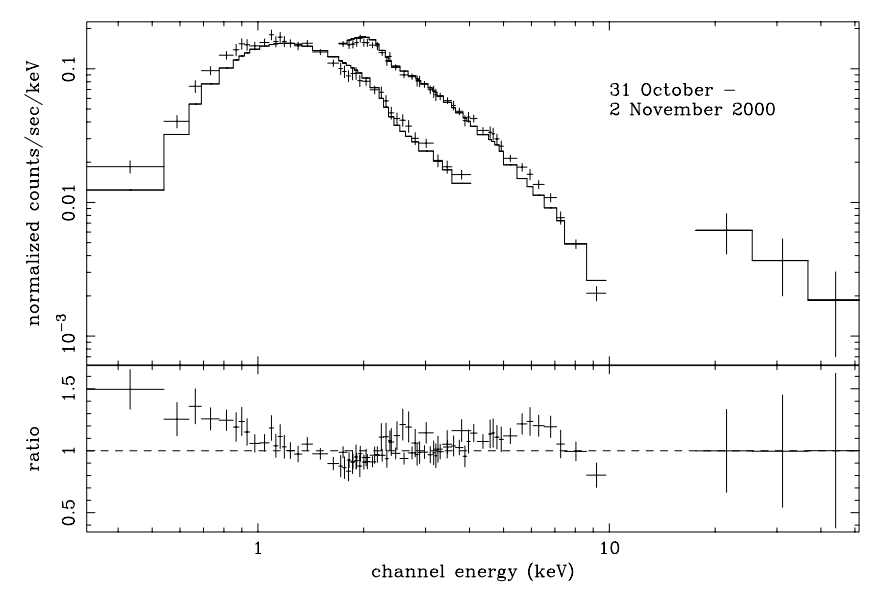

Fig. 8. LECS+MECS+PDS 31-October-2 November spectrum of BL Lac. fitted by the model in equation 9 , using $\Gamma_{1}=1$. If $\Gamma_{1} \leq 1$ the data are badly fitted by this model.

What we have proved to this point is that the bulk Compton can account for the X-ray data as long as the jet is pair rich. Further constraints about this emission mechanism come from the investigation of the pair loading processes occurring in the protojet, e.g. photon-photon interaction (Svensson 1987) or the interaction with the X-ray corona field (Sikora \& Madejski 2002), but this is beyond the goal of this paper.

However a simple viability test of the bulk Compton scenario can be performed which is based on the X-ray spectral shape. If this mechanism is working, in the X-ray band we should see the exponential tail of the blueshifted multitemperature blackbody emission of the accretion disk superposed to the usual hard inverse Compton emission. Therefore we tried to fit BeppoSAX data with a new model described by:

$$
A(v)=\mathrm{e}^{-N_{\mathrm{H}} \sigma(v)}\left(k_{1} v^{-\Gamma_{1}} \mathrm{e}^{-\left[\left(v_{0}-v\right) / v_{1}\right]}+k_{2} v^{-\Gamma_{2}}\right)
$$

where the second power law parameters are the best fit values obtained from modelling PDS data alone with a power law model $\left(\Gamma_{2}=1.76 ; k_{2}=3.19 \times 10^{-3}\right.$ photons $\left./ \mathrm{keV} / \mathrm{cm}^{2} / \mathrm{s}\right) ; N_{\mathrm{H}}$ was fixed to $2.5 \times 10^{21} \mathrm{~cm}^{-2}$. This model is not able to reproduce the data unless $\Gamma_{1}>1$ (see e.g. in Fig. 8 the residuals below $1 \mathrm{keV}$ ). This implies that a blueshifted Shakura-Sunyaev disk spectrum cannot reproduce the observed soft X-ray spectrum unless being characterized by a soft power law slope in the UV band.

\subsection{Two synchrotron components}

We considered then the hypothesis that the optical and the $\mathrm{X}$-ray spectra we observed are created by two populations of emitting electrons possibly located in two regions at different distances from the nucleus. One produces a synchrotron component peaking in the IR band and is responsible for the WEBT section while the second accounts for the BeppoSAX data. In order not to overproduce the optical flux, the X-ray component must peak above the UV band. This implies that the emitting particle population must have a break $\left(\gamma_{\text {break }}\right)$. If $N(\gamma) \propto \gamma^{-2}$ 
below $\gamma_{\text {break }}$, we require $\gamma_{\text {break }}>1.6 \times 10^{4} \delta_{10}^{-1 / 2} B^{-1 / 2}$ to not overproduce the optical flux.

The observed fast X-ray variability implies that the presence of a break in the particle distribution could not be an effect of the cooling. Since $t_{\mathrm{var}} \sim 6 \times 10^{3} \mathrm{~s}$ (see Table 4 ) the X-ray emitting region must be located at a distance from the nucleus $R \lesssim \delta c t_{\mathrm{var}} / \psi \sim 2 \times 10^{16} \Gamma_{10}^{2} \mathrm{~cm}$, where $\psi \sim 1 / \Gamma$ is the jet opening angle and $\delta=1 /\left(\Gamma\left(1-\beta \cos \theta_{\mathrm{obs}}\right)\right)$ is the Doppler factor. The dimension of the BLR of BL Lac is $R_{\mathrm{BLR}} \sim 8.3 \times 10^{16} M_{\mathrm{BH}, 8} \mathrm{~cm}$ (Böttcher \& Bloom 2000), where $M_{\mathrm{BH}, 8}$ is the black hole mass in units of $10^{8}$ solar masses. Since $M_{\mathrm{BH}, 8} \sim 1.7$ (Woo \& Urry 2002), $R_{\mathrm{BLR}} \sim 1.4 \times 10^{17} \mathrm{~cm}$ : the $\mathrm{X}$-ray emitting region should be inside the Broad Line Region, where the cooling rate is high.

If we assume that a continuous power law distribution of particles $N(\gamma) \propto \gamma^{-n}$ is injected for a time $t_{\mathrm{var}}$, then an electron above $\gamma_{\mathrm{c}}$ can cool in a time $t_{\mathrm{var}}$. After this time, above $\gamma_{\mathrm{c}}$ the particle population steepens to $N(\gamma) \propto \gamma^{-(n+1)}$, while below $\gamma_{\mathrm{c}}$ it remains unchanged. The value of $\gamma_{\mathrm{c}}$ is:

$$
\begin{aligned}
\gamma_{\mathrm{c}} & \sim \frac{3 m_{\mathrm{e}} c^{2}}{4 \sigma_{\mathrm{T}} c U_{\mathrm{BLR}}^{\prime}\left(1+U_{B}^{\prime} / U_{\mathrm{BLR}}^{\prime}+U_{\mathrm{s}}^{\prime} / U_{\mathrm{BLR}}^{\prime}\right) \delta t_{\mathrm{var}}} \\
& \sim 7 \times 10^{3} \frac{1}{\Gamma_{10}^{2} \delta_{10}}
\end{aligned}
$$

where we have used $U_{\mathrm{BLR}}^{\prime}=7 \times 10^{-2} \Gamma_{10}^{2} \mathrm{erg} \mathrm{cm}^{-3}$. We can see that $\gamma_{\mathrm{c}}$ is hardly consistent with the required $\gamma_{\text {break }}$, but, if during our observation the luminosity of the lines was fainter than the average $L_{\mathrm{BLR}}$ we have considered, then it is possible that $\gamma_{\mathrm{c}} \approx \gamma_{\text {break }}$. In this case the required break in the distribution can be accounted by the radiative cooling. Otherwise, not to overproduce the optical flux, the X-ray emitting particle distribution should be injected with an intrinsic break.

\subsection{Klein-Nishina effect on the synchrotron spectrum}

Since the Compton cooling rate of electrons with energies

$\gamma>\gamma_{1}=\frac{m_{\mathrm{e}} c^{2}}{\Gamma h v_{\mathrm{BEL}}} \simeq \frac{10^{4}}{\Gamma_{10} v_{\mathrm{BEL}, 15}}$

is reduced because of the decline of the Compton scattering cross section in the Klein-Nishina regime, for a given electron injection rate, the energy distribution of electrons hardens beyond $\gamma_{1}$. The effect is particularly strong for $f \equiv U_{\mathrm{BLR}}^{\prime} / U_{B}^{\prime} \gg 1$ and causes flattening of the synchrotron spectrum at

$$
\begin{aligned}
v>v_{1} & \simeq 3.6 \times 10^{6} \mathrm{~Hz} B \gamma_{1}^{2} \delta \\
& \simeq 5 \times 10^{15} \frac{\left(U_{\mathrm{BLR}} / 0.005 \mathrm{erg} \mathrm{cm}^{-3}\right)^{1 / 2}(\delta / \Gamma)}{(f / 10)^{1 / 2} v_{\mathrm{BEL}, 15}^{2}} \mathrm{~Hz} .
\end{aligned}
$$

The hardened part of the electron energy distribution extends up to $\gamma_{2} \sim f^{1 / 2} \gamma_{1}$. At $\gamma>\gamma_{2}$ the cooling of electrons becomes to be dominated by synchrotron radiation and the slope of the electron energy distribution becomes the same as at $\gamma \ll \gamma_{1}$. Consequently, at $v>v_{2} \sim f v_{1}$, the synchrotron spectrum steepens, regaining the slope from the optical band but vertically shifted up by a factor $f$ above the extrapolation of the optical spectral portion. Such a scenario can reproduce the observed spectral "glitch", provided $f \sim 10$. Details of the model will be presented elsewhere; here we would like to mention only that a similar scenario has been proposed by Dermer \& Atoyan (2002) to explain spectral glitches between the optical and $\mathrm{X}$-ray spectra of kiloparsec scale jets.

\section{Conclusions}

BL Lac has been the target of a multiwavelength campaign during 2000, extending from June to November. BeppoSAX observed the source twice, in July and at the end of October, while in different optical state of activity. As evidenced also from previous observations, BL Lac displays a very complex behaviour. We summarize the BeppoSAX results as follows:

- In July, while optically weak, the source displayed a faint, hard X-ray spectrum with positive residuals towards low energies. The soft $X$-ray flux varied in a timescale of a few hours, while the hard X-ray flux was almost constant.

- In October, while BL Lac was bright in the optical band, we observed in the $\mathrm{X}$-ray the transition from an extremely strong, soft component to a hard component dominating above $10-20 \mathrm{keV}$. The soft spectrum displayed an erratic temporal behaviour with large and fast variations on timescales down to $6 \times 10^{3} \mathrm{~s}$, while the hard component remained almost constant.

- BeppoSAX spectral results are confirmed by simultaneous RXTE data: PCA and MECS spectra are consistent within the uncertainties.

The frequency dependent temporal variability is consistent with the spectral analysis results: we observed fast and large flux variations when the soft component, interpreted as synchrotron emission, was dominating, while we observed constant light curves in the hard section of the spectra which can be reproduced as inverse Compton radiation in a leptonic jet model. This can be explained since synchrotron X-ray emitting electrons are more energetic than those that are producing inverse Compton emission at X-ray energies, so they cool faster. This behaviour was already observed in BL Lac (Ravasio et al. 2002) as well as in other similar objects, such as ON 231 (Tagliaferri et al. 2000) or S5 $0716+714$ (Tagliaferri et al. 2003).

The analysis of the multiwavelength Spectral Energy Distributions and the comparison with other historical SEDs, evidences the exceptionality of the X-ray spectrum of October 2000: during this observation BL Lac was displaying the highest soft X-ray flux ever recorded and an integrated [2-10] keV flux which was only sligthly smaller than that detected in July 1997, while BL Lac was in an exceptional flaring state and was displaying a hard X-ray spectrum (Madejski et al. 1999).

Moreover, the SED of October 2000 displayed another very interesting feature: the soft X-ray data laid above the extrapolation of the optical spectrum, while they should be both produced via the same synchrotron emission. To account for this inconsistency we have investigated 4 possibilities, among which, however, we cannot at present discriminate:

1. The dust-to-gas ratio towards BL Lac is higher than the interstellar one. However, since this misalignment is not seen 
in the other multiwavelength SED of BL Lac with a well defined synchrotron component (except that of 1980, where data have large uncertainties), a sudden large increase of the dust-to-gas ratio is needed. This hypothesis is not unlikely since dusty galactic nebulosities are indeed observed towards the source.

2. This is the first detection of the so called bulk Compton emission. In the hypothesis that the jet is pair loaded, we can reproduce the observed soft X-ray spectrum via the bulk Compton mechanism while the optical and the hard $\mathrm{X}$-ray spectra can be explained via the usual synchrotron and inverse Compton models. However, a blueshifted Shakura-Sunyaev disk spectrum cannot model BeppoSAX data. Nevertheless, we cannot exclude this scenario since the effective spectral shape of accretion disks in the far UV is still uncertain and could have a power law rather than an exponential slope (see e.g. Laor et al. 1996).

3. Two synchrotron components present at different distances from the nucleus account for the optical and the X-ray spectra, respectively. In order to not overproduce the optical flux and to account for the X-ray fast variability, the $\mathrm{X}$-ray emitting particle population must have a break at energies greater than $\gamma \sim 1.6 \times 10^{4} \delta_{10}^{-1 / 2} B^{-1 / 2}$. The break can be accounted by the particle cooling or must be intrinsic if the line luminosity level is lower or higher than the calculated average value, respectively.

4. Through the synchrotron emitting population, we observe the transition from inverse Compton cooling dominated to synchrotron cooling dominated particles. Between these two conditions, there is a range in which the electrons are dominated by an inefficient inverse Compton cooling in the Klein-Nishina regime: this produces a hardening in the particle population and therefore in the synchrotron spectrum which accounts for the observed optical to X-ray misalignment.

In the SED of October 2000 of BL Lac, above the synchrotron peak, we have detected a well determined misalignment between two sections of the synchrotron spectrum. To explain this feature, we suggested four scenarios presenting different favorable and critical points: at present, however, the theoretical models and the data do not allow us to discriminate between them, although the hypothesis of a variable dust-togas ratio is the most plausible one.

Acknowledgements. We are grateful to Dr. M. Villata for sending us informations about the optical data published in Villata et al. (2002). This research was financially supported by the Italian Ministry for University and Research. M.S. acknowledges partial support from Polish KBN grants: 5P03D00221 and 2P03C006 19p1,2.

\section{References}

Arbeiter, C., Pohl, M., \& Schlickeiser, R. 2002, A\&A, 386, 415

Blandford, R. D., \& Rees, M. J. 1978, in Pittsburgh Conference on BL Lac Objects, Pittsburgh, PA, April 24-26, ed. A. M. Wolfe (Pittsburgh: Univ. Pittsburgh Press), 328

Blazejowski, M., Sikora, M., Moderski, R., \& Madejski, G. M. 2000, ApJ, 545, 107
Bloom, S. D., Bertsch, D. L., Hartman, R. C., et al. 1997, ApJ, 490, L145

Boella, G., Butler, R. C., Perola, G. C., et al. 1997, A\&AS, 122, 299

Böttcher, M., \& Bloom, S. D. 2000, ApJ, 119, 469

Böttcher, M., Marscher, A. P., Ravasio, M., et al. 2003, in preparation Bradt, H. V., Rothschild, R. E., \& Swank, J. H. 1993, A\&AS, 97, 355

Bregman, J. N., Glassgold, A. E., Huggins, P. J., et al. 1990, ApJ, 352, 574

Cao, X. 2002, ApJ, 570, L13

Catanese, C. W., Akerlof, C. W., Biller, S. D., et al. 1997, ApJ, 480, 562

Celotti, A., \& Fabian, A. C. 1993, MNRAS, 264, 228

Celotti, A., Padovani, P., \& Ghisellini, G. 1997, MNRAS, 286, 415

Corbett, E. A., Robinson, A., Axon, D. J., et al. 1996, MNRAS, 281, 737

Corbett, E. A., Robinson, A., Axon, D. J., \& Hough, J. H., 2000, MNRAS, 311, 485

Denn, G. R., Mutel, R. L., \& Marscher, A. P. 2000, ApJS, 129, 61

Dermer, C. D., \& Atoyan, A. M. 2002, ApJ, 568, L81

Dermer, C. D., \& Schlickeiser, R. 1993, ApJ, 416, 458

Edelson, R. A., \& Krolik 1988, ApJ, 333, 646

Edelson, R. A. 1992, ApJ, 401, 516

Elvis, M., Lockman, F. J., \& Wilkes, B. J. 1989, AJ, 97, 777

Fiore, F., Guainazzi, M., \& Grandi, P. 1999, Cookbook for NFI BeppoSAX Spectral Analysis v.1.2

Fossati, G., Maraschi, L., Celotti, A., Comastri, A., \& Ghisellini, G. 1998, MNRAS, 299, 433

Fossati, G., Celotti, A., Chiaberge, M., et al. 2000, ApJ, 541, 153

Ghisellini, G., \& Madau, P. 1996, MNRAS, 280, 67

Ghisellini, G., Celotti, A., Fossati, G., Maraschi, L., \& Comastri, A. 1998, MNRAS, 301, 451

Ghisellini, G. 2000, Blazar Demographics and Physics, ASP Conf. Ser., 227, 85

Ghisellini, G., Celotti, A., \& Costamante, L. 2002, A\&A, 386, 833

Giommi, P., \& Fiore, F. 1998, in The 5th International Workshop on Data Analysis in Astronomy, Erice, Italy, ed. V. Di Gesú, M. J. B. Duff, A. Heck, et al. (Word Scient. Publ.), 73

Jahoda, K., Swank, J. H., Giles, A. B., et al. 1996, Proc. SPIE, 2808, 59

Kawai, N., Matsuoka, M., Bregman, J. N., et al. 1991, ApJ, 382, 508

Laor, A., Fiore, F., Elvis, M., Wilkes, B. J., \& McDowell, J. C. 1997, ApJ, 477, 93

Lucas, R., \& Liszt, H. S. 1993, A\&A, 176, L33

Madejski, G. M., Sikora, M., Jaffe, T., et al. 1999, ApJ, 521, 145

Mang, O., Schilling, M., Siems, M., et al. 2001, in Proc. of the 27th ICRC, 2658

Maraschi, L., Ghisellini, G., \& Celotti, A. 1992, ApJ, 397, L5

Marscher, A. P., et al., in preparation

Miller, J. S., \& Hawley, S. A. 1977, ApJ, 212, L47

Miller, J. S., French, H. B., \& Hawley, S. A. 1978, ApJ, 219, L85

Mutel, R. L., Phillips, R. B., Su, B., \& Bucciferro, R. R., 1990, ApJ, 352,81

Padovani, P., \& Giommi, P. 1995, ApJ, 444, 567

Padovani, P., Costamante, L., Giommi, P., et al. 2001, MNRAS, 328, 931

Parmar, A. N., Oosterbroek, T., Orr, A., et al. 1999, A\&AS, 122, 309

Penston, M. V., \& Penston, M. F. 1973, MNRAS, 162, 109

Ravasio, M., Tagliaferri, G., Ghisellini, G., et al. 2002, A\&A, 383, 763

Ryter, C. E. 1996, Ap\&SS, 236, 285

Sambruna, R. M., Ghisellini, G., Hooper, E., et al. 1999, ApJ, 515, 140

Sambruna, R. M., Maraschi, L., \& Urry, M. C. 1996, ApJ, 463, 444

Schlegel, D. J., Finkbeiner, D. P., \& Davis, M. 1998, ApJ, 500, 525 
Schmitt, J. 1968, Nature, 218, 663

Sikora, M., Begelman, M. C., \& Rees, M. J. 1994, ApJ, 421,153

Sikora, M., Madejski, G., Moderski, R., \& Poutanen, J. 1997, ApJ, 484, 108

Sikora, M., \& Madejski, G. 2002, ApJ, 534, 109

Sillanpää, A., Takalo, L. O., Nilsson, K., \& KiKuchi, S. 1993, Ap\&SS, 206, 55

Simonetti, J. H., Cordes, J. M., \& Heeschen, D. S. 1985, ApJ, 196, 46 Spada, M., Ghisellini, G., Lazzati, D., \& Celotti, A. 2001, MNRAS, 325, 1559

Svensson, R. 1987, MNRAS, 227, 403
Tagliaferri, G., Ghisellini, G., et al. 2000, A\&A, 354, 431

Tagliaferri, G., Ravasio, M., Ghisellini, G., et al. 2003, A\&A, 400, 477 Urry, C. M., Sambruna, R. M., Worrall, D. M., et al. 1996, ApJ, 463, 424

Vermeulen, R. C., Ogle, P. M., Tran, H. D., et al. 1995, ApJ, 452, L5

Villata, M., Raiteri, C. M., Kurtanidze, O. M., et al. 2002, A\&A, 390, 407

Woo, J. H., \& Urry, M. C. 2002, ApJ, 579, 530

Worrall, D. M., \& Wilkes, B. J. 1990, ApJ, 360, 396

Wu, X. B., Liu, F. K., \& Zhang, T. Z. 2002, A\&A, 389, 742

Zhang, Y. H., Celotti, A., Treves, A., et al. 1999, ApJ, 527, 719 